\title{
A Nullclines Approach to the Study of 2D Artificial Network
}

\section{Felix Sadyrbaev ${ }^{1}$, Diana Ogorelova ${ }^{2}$, Inna Samuilik ${ }^{2}$}

${ }^{1}$ Institute of Mathematics and Computer Science, University of Latvia, Riga, Latvia

${ }^{2}$ Faculty of Natural Sciences and Mathematics, Daugavpils University, Daugavpils, Latvia E-mail: felix@latnet.lv

Abstract: The system of two the first-order ordinary differential equations arising in the gene regulatory networks theory is studied. The structure of attractors for this system is described in three important behavioral cases: activation, inhibition, mixed activation-inhibition. The geometrical approach combined with the vector field analysis allows treating the problem in full generality. A number of propositions are stated and the proof is geometrical, avoiding complex analytic. Although not all the possible cases are considered, the instructions are given on what to do in any particular situation.

Keywords: gene regulatory networks, phase portraits, bifurcations, critical points, attractors

\section{Introduction}

The system ${ }^{[1]}$

$$
x_{i}^{\prime}=f_{i}(x)-\gamma_{i} x_{i}
$$

is used to model the behavior of some artificial networks including the gene regulatory networks (GRN in short). Functions $f_{i}(z)$ are sigmoidal functions, that is, each $f_{i}(x)$ is monotonically increasing from zero to unity as the argument $z$ goes from $-\infty$ to $+\infty$ and, moreover, the graph of $f_{i}(z)$ has only one point of inflexion. The typical sigmoidal functions are the logistic function ${ }^{[2]}$

$$
f(z)=\frac{1}{1+e^{-\mu(z-\theta)}}
$$

and Gompertz function ${ }^{[3]}$

$$
f(z)=e^{-e^{-\mu(z-\theta)}},
$$

where $\mu$ and $\theta$ are parameters. For this particular choice of $f$ the system (1) in the extended form is

$$
\left\{\begin{array}{c}
\frac{d x_{1}}{d t}=\frac{1}{1+e^{-\mu_{1}\left(w_{11} x_{1}+w_{12} x_{2}+\ldots+w_{1 n} x_{n}-\theta_{1}\right)}}-\gamma_{1} x_{1}, \\
\frac{d x_{2}}{d t}=\frac{1}{1+e^{-\mu_{2}\left(w_{21} x_{1}+w_{22} x_{2}+\ldots+w_{2 n} x_{n}-\theta_{2}\right)}}-\gamma_{2} x_{2}, \\
\ldots \\
\frac{d x_{n}}{d t}=\frac{1}{1+e^{-\mu_{n}\left(w_{n 1} x_{1}+w_{n 2} x_{2}+\ldots+w_{n n} x_{n}-\theta_{n}\right)}}-\gamma_{n} x_{n} .
\end{array}\right.
$$


A similar expansion is valid for the Gompertz function.

System (4) is a simplified form of GRN.

GRN can be thought of as complex dynamical systems that are present in living organisms (on a cellular level) and which are constantly changing their states responding to fluctuations in their environment. The interaction of elements in living cells strongly depends on the interrelation of genes encoding proteins as transcription factors. In the above system the variables $x_{i}$ stand for the protein concentrations. Each $x_{i}$ is affected by other elements $x_{j},(j \neq i)$ of a network. The cumulative impact on $x_{i}$ is recorded as the linear combination $w_{i 1} x_{1}+w i_{2} x_{2}$ $+\ldots+w_{i n} x_{n}$ in the argument of $f_{i}$. The element $x_{\mathrm{i}}$ begins to respond to the total effect of the other elements by the expression of proteins when this effect reaches a threshold $\theta_{i}$. The ability of $x_{i}$ to respond to the effects of other elements is expressed by the parameter $\mu_{i}$. The coefficients $w_{i j}$ in the system (4) can be gathered in the matrix

$$
W=\left(\begin{array}{cccc}
w_{11} & w_{12} & \ldots & w_{1 n} \\
w_{21} & w_{22} & \ldots & w_{2 n} \\
\ldots & & & \\
w_{n 1} & w_{n 2} & \ldots & w_{n n}
\end{array}\right)
$$

which is called the regulatory matrix. A positive coefficient $w_{i j}$ means that $x_{\mathrm{j}}$ activates the expression of protein $x_{i}$, while the negative coefficient means the inhibition (the repression). The zero coefficient means no relation. $[9],[8]$.

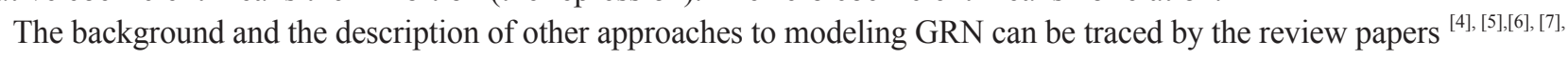

The other approaches include graph theory, Boolean algebras, stochastic functions, etc. It is acknowledged that one of the more effective tools for studying GRN and their evolution is the differential equations approach. Indeed, imagine that the current state of a given GRN is described by the vector $\left(x_{1}(t), \ldots, x_{n}(t)\right)$. The phase space $R^{n}$ contains information about possible scenarios of evolution. The future of the system is described by the attracting sets. In simple models, these sets are merely stable critical points and/or limit cycles ${ }^{[10],[1]]}$.

The study of attractors of a general system is not an easy problem. Even the definition of an attractor is a matter of discussion. The minimal GRN consists of two equations and the corresponding system of differential equations is

$$
\left\{\begin{array}{l}
x_{1}^{\prime}=\frac{1}{1+e^{-\mu_{1}\left(w_{11} x_{1}+w_{12} x_{2}-\theta_{1}\right)}}-\gamma_{1} x_{1}, \\
x_{2}^{\prime}=\frac{1}{1+e^{-\mu_{2}\left(w_{21} x_{1}+w_{22} x_{2}-\theta_{2}\right)}}-\gamma_{2} x_{2} .
\end{array}\right.
$$

The dynamics of this system can be explored by the phase-plane analysis in particular cases. The global study is not easy since the system contains ten parameters. Bifurcations can be studied for restricted small groups of parameters. We mention the work by K.W. Kohn ${ }^{[12]}$, who studied the system

$$
\left\{\begin{array}{l}
x_{1}^{\prime}=k_{1} h\left(x_{2}, \theta_{2}, \mu_{2}\right)-\gamma_{1} x_{1}, \\
x_{2}^{\prime}=k_{2} h\left(x_{1}, \theta_{1}, \mu_{1}\right)-\gamma_{2} x_{2},
\end{array}\right.
$$

where $h(z, \theta, \mu)$ is the (sigmoidal) Hill's function

$$
h(z)=\frac{z^{\mu}}{z^{\mu}+\theta^{\mu}},
$$

that is increasing in the case of activation, or 


$$
h(z)=\frac{\theta^{\mu}}{z^{\mu}+\theta^{\mu}}
$$

in the case of inhibition (the decreasing function). In the paper ${ }^{[13]}$ bifurcations in the system (22) were studied provided that $\mu_{1}=\mu_{2}, \theta_{1}=\theta_{2}, w_{11}=w_{22}=0, w_{12}=w_{21}=1$. In a series of recent publications ${ }^{[14]-[18]}$ particular systems of lowdimensions were investigated.

In the work ${ }^{[19]}$ the $n$-dimensional system (4) was studied for specific regulatory matrices $W$. We refer also to the works ${ }^{[20]-[22]}$, where similar techniques were used.

We provide a simple geometrical approach via the phase plane consideration for two dimensional systems of the form

$$
\left\{\begin{array}{l}
x_{1}^{\prime}=\frac{1}{1+e^{-\mu_{1}\left(w_{11} x_{1}+w_{12} x_{2}-\theta_{1}\right)}}-\gamma_{1} x_{1}, \\
x_{2}^{\prime}=\frac{1}{1+e^{-\mu_{2}\left(w_{21} x_{1}+w_{22} x_{2} \theta_{2}\right)}}-\gamma_{2} x_{2},
\end{array}\right.
$$

and/or

$$
\left\{\begin{array}{c}
x_{1}^{\prime}=\exp \left(-\exp \left[-\mu_{1}\left(w_{11} x_{1}+w_{12} x_{2}-\theta_{1}\right)\right]\right)-\gamma_{1} x_{1}, \\
x_{2}^{\prime}=\exp \left(-\exp \left[-\mu_{2}\left(w_{21} x_{1}+w_{22} x_{2}-\theta_{2}\right)\right]\right)-\gamma_{2} x_{2},
\end{array}\right.
$$

which allows an overview of multiple variants of the behavior. All we use is the nullclines method, analysis of the vector field defined by the system, and some knowledge about the dependence of a set of solutions on the parameters of the system. We consider three types of behaviors, which are called activation, inhibition, and activation-inhibition cases. This is justified by the above explanation of the meaning of elements of the matrix $W$. The matrix $W$ for our treatment is

$$
W=\left(\begin{array}{ll}
w_{11} & w_{12} \\
w_{21} & w_{22}
\end{array}\right)
$$

Our goal is to describe the behavior of attractors (sets of critical points) for the system (10). Our approach allows doing this without considering multiple parameters.

\section{General}

We assume that $\mu_{1}$ and $\mu_{2}$ are positive, as well as $\gamma_{1}$ and $\gamma_{2}$.

First consider the nullclines of the system (10) which are given by

$$
\left\{\begin{array}{l}
x_{1}=\frac{1}{\gamma_{1}} \frac{1}{1+e^{-\mu_{1}\left(w_{11} x_{1}+w_{12} x_{2}-\theta_{1}\right)}}, \\
x_{2}=\frac{1}{\gamma_{2}} \frac{1}{1+e^{-\mu_{2}\left(w_{21} x_{1}+w_{22} x_{2}-\theta_{2}\right)}} .
\end{array}\right.
$$

Introduce the functions

$$
f_{1}\left(x_{1}, x_{2}\right)=\frac{1}{1+e^{-\mu_{1}\left(w_{11} x_{1}+w_{12} x_{2}-\theta_{1}\right)}}, \quad f_{2}\left(x_{1}, x_{2}\right)=\frac{1}{1+e^{-\mu_{2}\left(w_{21} x_{1}+w_{22} x_{2}-\theta_{2}\right)}}
$$

and similar functions for the Gompertz case 


$$
\begin{aligned}
& g_{1}\left(x_{1}, x_{2}\right)=\exp \left(-\exp \left[-\mu_{1}\left(w_{11} x_{1}+w_{12} x_{2}-\theta_{1}\right)\right]\right), \\
& g_{2}\left(x_{1}, x_{2}\right)=\exp \left(-\exp \left[-\mu_{2}\left(w_{21} x_{1}+w_{22} x_{2}-\theta_{2}\right)\right]\right) .
\end{aligned}
$$

Since the range of values of sigmoidal functions is the interval $(0,1)$, the nullclines defined by $(14)$ are located in the strips $0<x_{1}<\frac{1}{\gamma_{1}}$ and $0<x_{2}<\frac{1}{\gamma_{2}}$ respectively. Therefore the first assertion.

Proposition 2.1 All the critical points of the system (10) (or system (11)) are located in the rectangle $Q=\left(0, \frac{1}{\gamma_{1}}\right) \times\left(0, \frac{1}{\gamma_{2}}\right)$. There is at least one critical point.

Proof. The critical points are cross points of the nullclines (13). These nullclines have points in common only in the rectangle $Q$ They must intersect in $Q$.

Proposition 2.2 The vector field $\left(x_{1}^{\prime}, x_{2}^{\prime}\right)$, defined by (10) (or by (11)), is directed inward on the border of the rectangle Q.

Proof. By inspection of the right-hand sides in (10), taking into account (13) and the range of values of the functions $f_{1}(z)$ and $f_{2}(z)$.

Corollary 2.1 Any trajectory of the system (10) (or of the system (11)), entering $Q$ will stay there for $t>0$

Proposition 2.3 There are at most three critical points for the system (10)(or for the system (11)) if $w_{11}=w_{22}=0$. Exactly one and exactly two critical points are possible. $w_{12}=\alpha, w_{21}=\beta$ has not closed orbits in $Q$ if $w_{11}=w_{22}=0$.

Proof. The divergence of the vector field, defined by (10), is

$$
\frac{\partial\left\{\frac{1}{1+e^{-\mu_{1}\left(\alpha x_{2}-\theta_{1}\right)}}-\gamma_{1} x_{1}\right\}}{\partial x_{1}}+\frac{\partial\left\{\frac{1}{1+e^{-\mu_{2}\left(\beta x_{1}-\theta_{2}\right)}}-\gamma_{2} x_{2}\right\}}{\partial x_{2}}=-\gamma_{1}-\gamma_{2} \neq 0 .
$$

By the Bendixson criterium ${ }^{[23]}$, there are no closed orbits in $Q$. Proof for the system (11) is similar.

\section{The nullclines analysis for Gompertz function}

\subsection{Activation}

Consider the system (11) where $w_{11}=w_{22}=0$, and $w_{12}=\alpha, w_{21}=\beta, \gamma_{1}, \gamma_{2}$ are positive. This corresponds to the activation case. The nullclines

$$
\left\{\begin{array}{l}
x_{1}=\frac{1}{\gamma_{1}} \exp \left(-\exp \left[-\mu_{1}\left(w_{11} x_{1}+w_{12} x_{2}-\theta_{1}\right)\right]\right) \\
x_{2}=\frac{1}{\gamma_{2}} \exp \left(-\exp \left[-\mu_{2}\left(w_{21} x_{1}+w_{22} x_{2}-\theta_{2}\right)\right]\right)
\end{array}\right.
$$

for three possible cases are depicted in Fig. 9 to Fig. 11. We set $\alpha=1, \beta=2, \gamma_{1}=\gamma_{2}=1$.
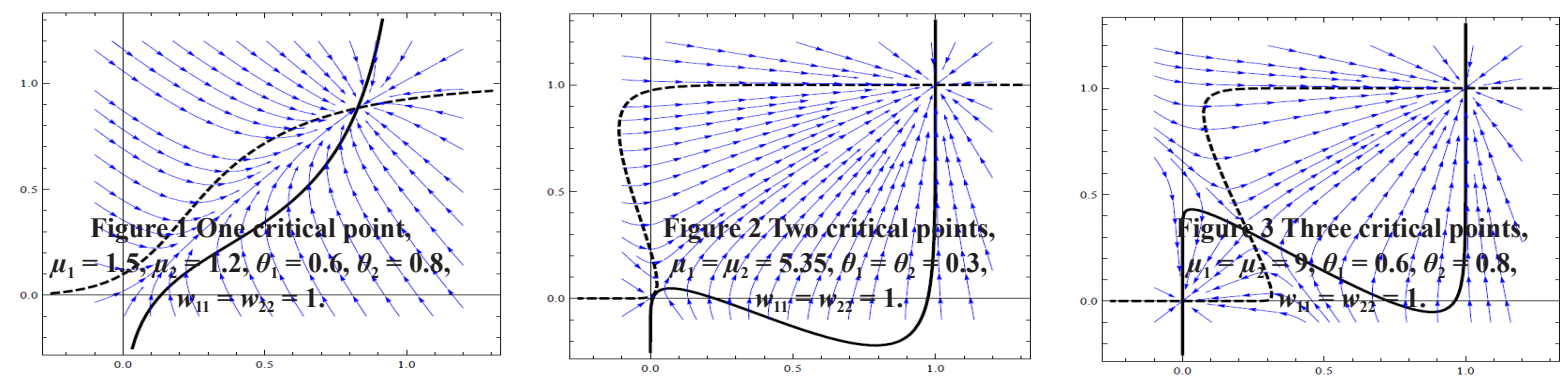
The first case corresponds to the nullclines transversally intersecting in a unique critical point. The vector field $\left(x_{1}^{\prime}, x_{2}^{\prime}\right)$ is directed towards the critical point which is a sink.

The second case is visualized in Fig. 2. Two nullclines intersect transversally and have a tangent point also. The upper (simple) critical point is a sink. Consider the lower critical point. Let it be at $\left(z_{1}, z_{2}\right)$. Linearization of the system (10) at $\left(z_{1}\right.$, $z_{2}$ ) yields the linear system

$$
\left\{\begin{array}{l}
u_{1}^{\prime}=-u_{1}+f_{1}^{\prime}\left(z_{2}\right) u_{2}, \\
u_{2}^{\prime}=f_{2}^{\prime}\left(z_{1}\right) u_{1}-u_{2},
\end{array}\right.
$$

where $f_{1}$ and $f_{2}$ are defined in (14). One has at the point $\left(z_{1}, z_{2}\right)$ that $\frac{d x_{1}}{d x_{2}}=f_{1}^{\prime}\left(z_{2}\right), \frac{d x_{2}}{d x_{1}}=f_{2}^{\prime}\left(z_{1}\right)$. Since both nullclines are tangent at this point one has that $\frac{d x_{2}}{d x_{1}}$ are equal and

$$
\frac{d x_{2}}{d x_{1}}=\frac{1}{f_{1}^{\prime}\left(z_{2}\right)}=f_{2}^{\prime}\left(z_{1}\right), \text { or } f_{1}^{\prime}\left(z_{2}\right) f_{2}^{\prime}\left(z_{1}\right)=1 \text {. }
$$

The characteristic equation for the system $(17)$ is $(\lambda+1)^{2}=f_{1}^{\prime}\left(z_{2}\right) f_{2}^{\prime}\left(z_{1}\right)=1$ and the characteristic roots are $\lambda_{1}=0, \lambda_{2}$ $=-2$. The point $\left(z_{1}, z_{2}\right)$ is degenerate therefore and the trajectory tangent to nullclines is directed away from the point $\left(z_{1}\right.$, $z_{2}$ ). The location of the tangent point above the simple one is also possible. The conclusion is the same, namely, the simple critical point is a sink, the tangent critical point has an escape direction.

There are three critical points in the third case and two of them are sinks. The central point is a saddle. The summary of this section follows.

Proposition 3.1 The attractor of the system (10), where $\alpha$ and $\beta$ are positive, can consist of either one or two stable critical points.

There is also an exceptional case where nullclines have a tangent point of order two (both the first and the secondorder derivatives $\frac{d x_{2}}{d x_{1}}$ are equal at the critical point. This point is attractive as shown in Fig.4.

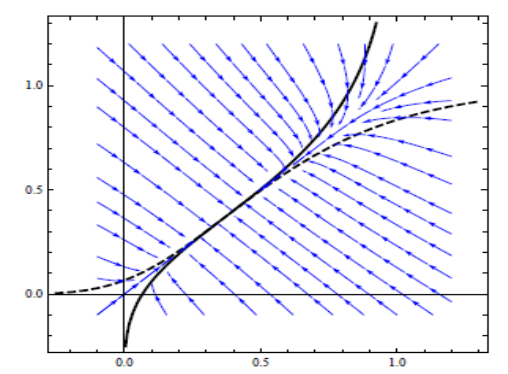

Figure 4 Exceptional case, $\mu_{1}=\mu_{2}=\mathrm{e}, \theta_{1}=\theta_{2}=1 / \mathrm{e}, \alpha=\beta=1, w_{11}=w_{22}=0$.

\subsection{Mixed activation-inhibiton}

Consider the system (10) where the signs of $\alpha$ and $\beta$ are opposite. The nullclines together with the vector field are depicted in Fig. 5. If $\alpha$ is positive then the rotation of the vector field is in the counter clock-wise direction. 


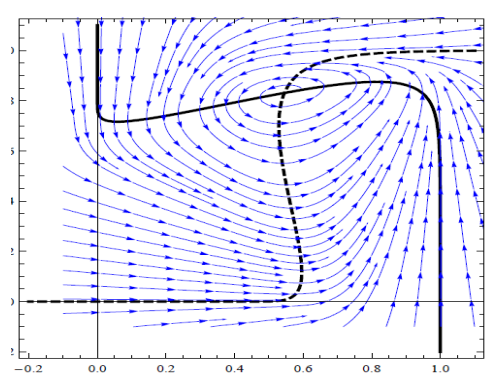

Figure 5 One critical point, $\alpha=-2, \beta=2, \mu_{1}=6, \mu_{2}=4$, $\theta_{1}=-1.2, \theta_{2}=1.5, w_{11}=w_{22}=1$.

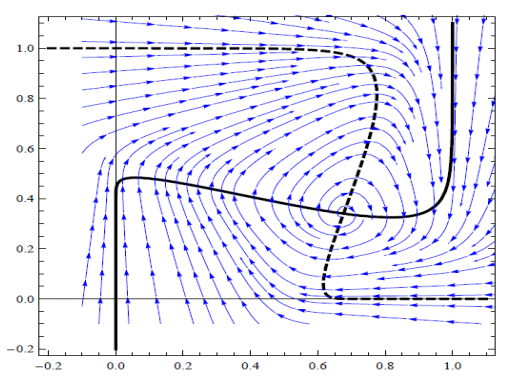

Figure 6 One critical point,

$\alpha=2, \beta=-2, \mu_{1}=\mu_{2}=6$, $\theta_{1}=1.2, \theta_{2}=-1.0, w_{11}=w_{22}=1$.

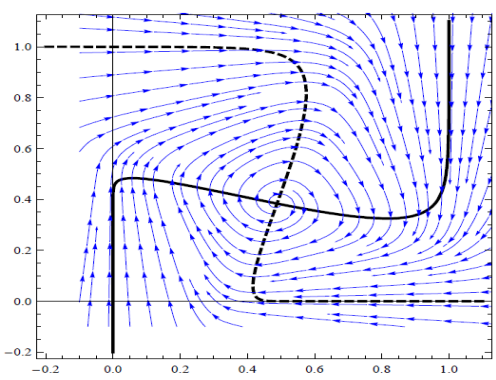

Figure 7 One critical point, $\alpha=2, \beta=-2, \mu_{1}=\mu_{2}=6$, $\theta_{1}=1.2, \theta_{2}=-0.6, w_{11}=w_{22}=1$. The closed trajectory (limit cycle) is indicated by pointer.

If $\alpha$ is negative and $\beta$ positive then (Fig. 6) the rotation of vector field is in the clock-wise direction.

Proposition 3.2 There is a single critical point of the type stable focus for the system (10) if $\alpha$ and $\beta$ have opposite signs.

Proof. Suppose for definiteness that $\alpha$ is positive and $\beta$ is negative. The critical points are solutions of the system (13) or

$$
\left\{\begin{array}{l}
x_{1}=\frac{1}{\gamma_{1}} f_{1}\left(x_{2}\right), \\
x_{2}=\frac{1}{\gamma_{2}} f_{2}\left(x_{1}\right),
\end{array}\right.
$$

where the functions $f_{1}$ and $f_{2}$ are defined in (14). The function $f_{1}\left(x_{2}\right)$ is increasing together with the inverse function $x_{2}=$ $f_{1}^{-1}(x 1)$. The function $x_{2}=\frac{1}{\gamma_{2}} f_{2}\left(x_{1}\right)$ is decreasing. Therefore the system (which is equivalent to the system (19))

$$
\left\{\begin{array}{l}
x_{2}=f_{1}^{-1}\left(\gamma_{1} x_{1}\right), \\
x_{2}=\frac{1}{\gamma_{2}} f_{2}\left(x_{1}\right),
\end{array}\right.
$$

has only one solution $\left(x_{1}, x_{2}\right)$.

In order to detect the type of the critical point consider the linearized system

$$
\left\{\begin{array}{l}
u_{1}^{\prime}=-u_{1}+\frac{1}{\gamma_{1}} f_{1}^{\prime}\left(x_{2}\right) u_{2}, \\
u_{2}^{\prime}=\frac{1}{\gamma_{2}} f_{2}^{\prime}\left(x_{1}\right) u_{1}-u_{2},
\end{array}\right.
$$

Notice that $f_{1}^{\prime}\left(x_{2}\right)$ is negative and $f_{2}^{\prime}\left(x_{1}\right)$ is positive. The characteristic equation for the critical point $\left(x_{1}, x_{2}\right)$ is

$$
(\lambda+1)^{2}=\frac{1}{\gamma_{1} \gamma_{2}} f_{1}^{\prime}\left(x_{2}\right) f_{2}^{\prime}\left(x_{1}\right)
$$

and the characteristic roots are 


$$
\lambda_{1,2}=-1 \pm \sqrt{\frac{1}{\gamma_{1} \gamma_{2}} f_{1}^{\prime}\left(x_{2}\right) f_{2}^{\prime}\left(x_{1}\right)} .
$$

Since the real part is negative and the expression under the square root sign is also negative, the critical point $\left(x_{1}, x_{2}\right)$ is a stable focus. The proof for the case of $\alpha$ negative and $\beta$ positive is similar.

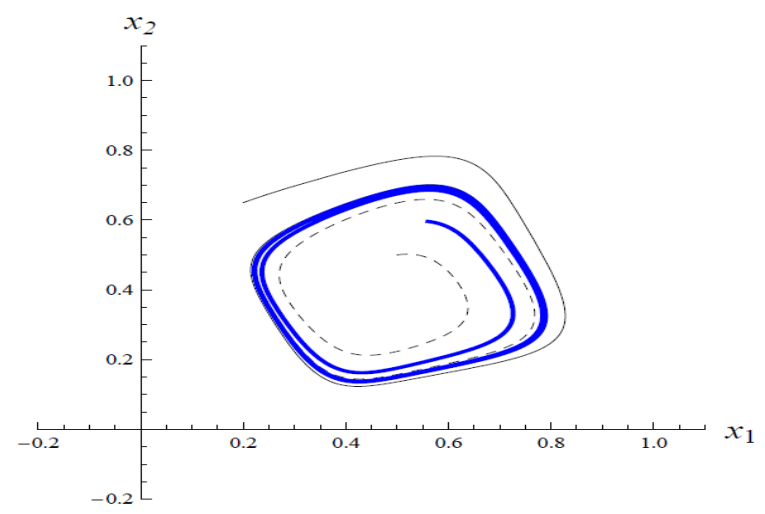

Figure 8 The limit cycle passing through the point $(0.49,0.38)$, $w_{11}=w_{22}=1, \alpha=2, \beta=-2, \mu_{1}=6, \mu_{2}=6, \theta_{1}=1.2, \theta_{2}=-0.6$.

Remark. Proposition 2.4 says that there are no closed orbits in the system (10) if $w_{11}=w_{22}=0$. If $w_{11}$ and $w_{22}$ are allowed to be nonzero, the limit cycle is possible. In Fig. 7 the limit cycle appears as the result of Hopf bifurcation when the unstable focus arises. A vicinity of the unstable focus at $\left(x_{1}, x_{2}\right)$ together with the boundary of the rectangle $\left\{\left(y_{1}, y_{2}\right): 0<y_{1}<\frac{1}{\gamma_{1}}, 0<y_{2}<\frac{1}{\gamma_{2}}\right\}$ form the Bendixson region ${ }^{[23]}$ with the vector field on the boundary directed inward and with no critical points in the interior.

\section{The nullclines analysis for logistic function}

In this section, we change the Gompertz function to the logistic function (both are sigmoidal) and allow the diagonal elements of the regulatory matrix $W$ to be nonzero. In a series of examples, we show that the shape of nullclines may change substantially and as the result, the number of critical points and their location is different compared with those in the preceding section.

\subsection{The parameter $\mu$ is small}

We consider the system

$$
\left\{\begin{array}{c}
x_{1}^{\prime}=\frac{1}{1+e^{-\mu_{1}\left(w_{11} x_{1}+w_{12} x_{2}-\theta_{1}\right)}}-\gamma_{1} x_{1}, \\
x_{2}^{\prime}=\frac{1}{1+e^{-\mu_{2}\left(w_{21} x_{1}+w_{22} x_{2}-\theta_{2}\right)}}-\gamma_{2} x_{2} .
\end{array}\right.
$$

Set the parameters $\mu_{1}=\mu_{2}=2, \theta_{1}=\theta_{2}=0.1, \gamma_{1}=\gamma_{2}=1$, but the entries of the regulatory matrix $W$ will be flexible. The following choices of $W$ will be considered. 

1. $W=\left(\begin{array}{ll}4 & 2 \\ 3 & 5\end{array}\right)$
2. $W=\left(\begin{array}{cc}-4 & 2 \\ 3 & 5\end{array}\right)$
3. $W=\left(\begin{array}{cc}4 & -2 \\ 3 & 5\end{array}\right)$
4. $W=\left(\begin{array}{cc}4 & 2 \\ -3 & 5\end{array}\right)$
5. $W=\left(\begin{array}{cc}4 & 2 \\ 3 & -5\end{array}\right)$

By changing values of $w_{12}$ and $w_{21}$ we consider several cases of possible regulatory matrices. Set $\mu=2, \theta=0.1$, but the entries of the matrix $W$ will be changed.

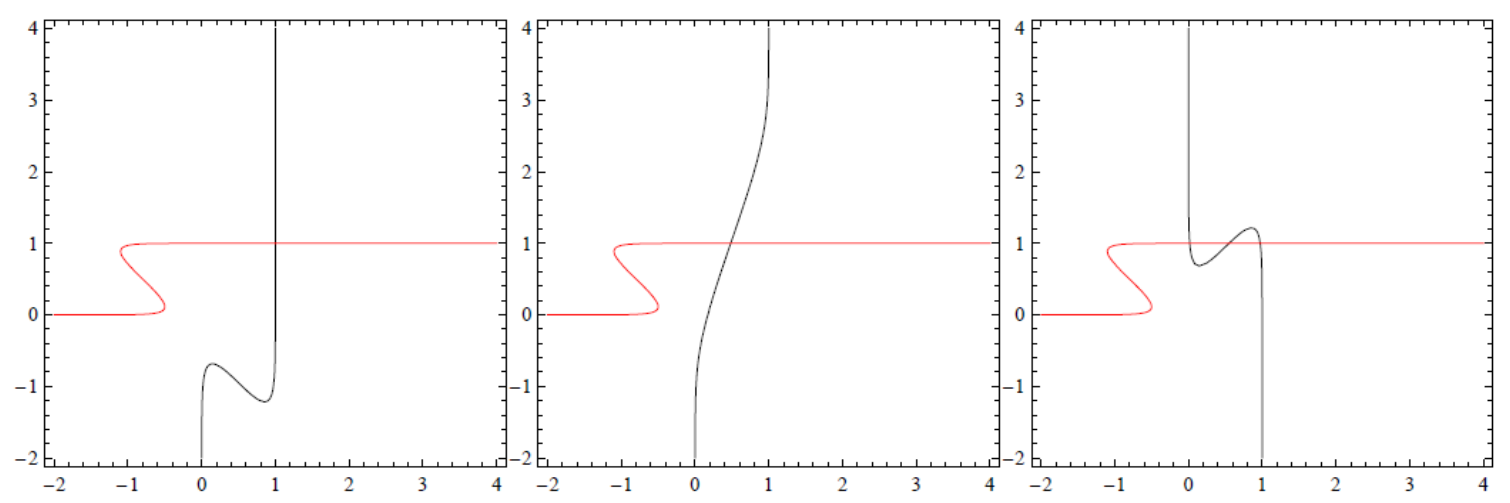

(a)

(b)

(c)

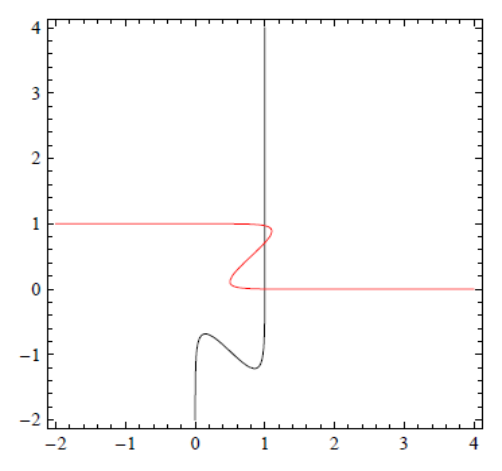

(d)

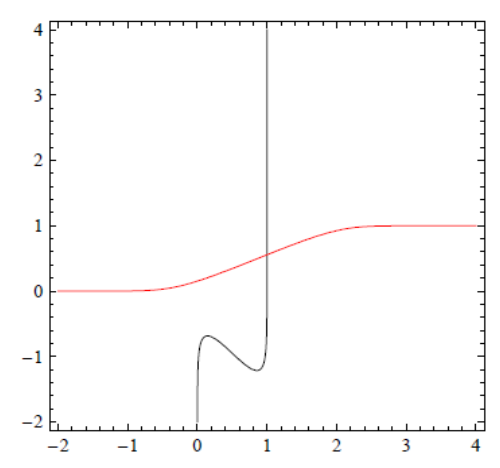

(e)

Figure 9 (a) $\mathrm{w}_{11}=4, \mathrm{w}_{12}=2, \mathrm{w}_{21}=3, \mathrm{w}_{22}=5$; (b) $w_{11}=-4, w_{12}=2, w_{21}=3, w_{22}=5$; (c) $w_{11}=4, w_{12}=-2, w_{21}=3, w_{22}=5$; (d) $w_{11}=4, w_{12}=2, w_{21}=-3, w_{22}=5$;

(e) $w_{11}=4, w_{12}=2, w_{21}=3, w_{22}=-5$

\subsection{The parameter $\mu$ is large}

Let us consider the example with $\mu=40, \theta_{1}=\theta_{2}=3.5, \gamma_{1}=\gamma_{2}=1$, but the entries of the matrix $W$ are $w_{11}=4, w_{12}=$ $2, w_{21}=3$ and $w_{22}=5$. The graph of sigmoid functions is "Z-shaped" curve and this system has nine critical points (see Fig.10). 


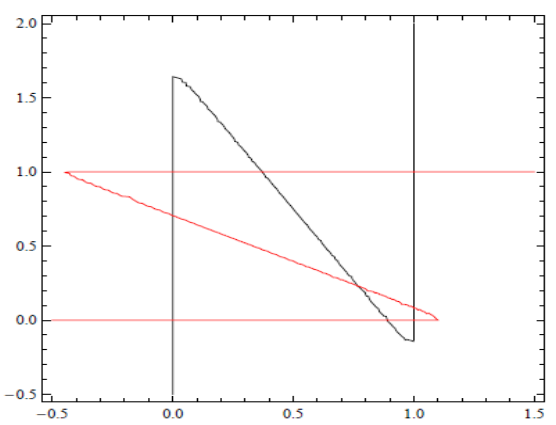

Figure 10 The graph of sigmoid functions is "Z-shaped" curve and this system has nine critical points

Let $\mu=40, \gamma_{1}=\gamma_{2}=1$ and the entries of the matrix $\mathrm{W}$ are $w_{11}=9, w_{12}=6, w_{21}=2, w_{22}=3$.

Proposition 4.1 Five critical points are possible for the system (22).

Proof. By construction the example (Fig. 11(a) and the given values of parameters).

Proposition 4.2 Seven critical points are possible for the system (22).

Proof. By construction the example (Fig. 11(b) and the given values of parameters).

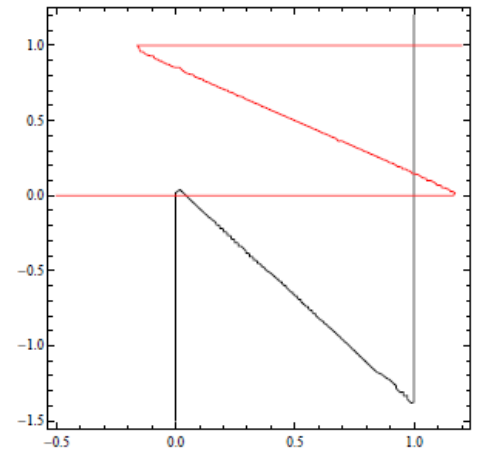

(a)

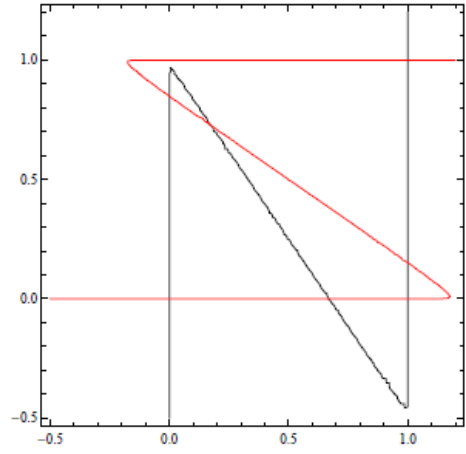

(b)

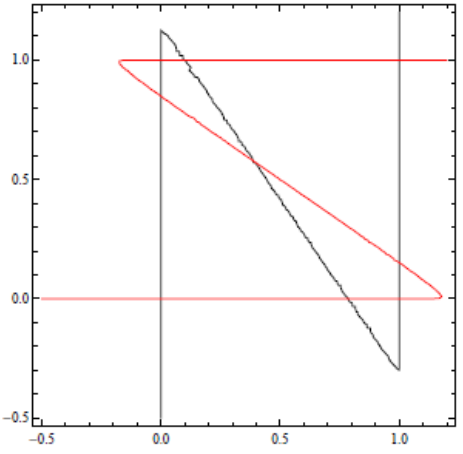

(c)

Figure 11 (a) $\theta_{1}=0.5, \theta_{2}=2.5$; (b) $\theta_{1}=6, \theta_{2}=2.5 ;$ (c) $\theta_{1}=7, \theta_{2}=2.5$

Proposition 4.3 The maximal number of critical points is nine for the system (22).

Proof. By construction the example (Fig. 11(c) and the given values of parameters).

Let $\mu=40, \gamma_{1}=\gamma_{2}=1$ and the entries of the matrix $\mathrm{W}$ are $w_{11}=9, w_{12}=6, w_{21}=-2$ and $w_{22}=3$.

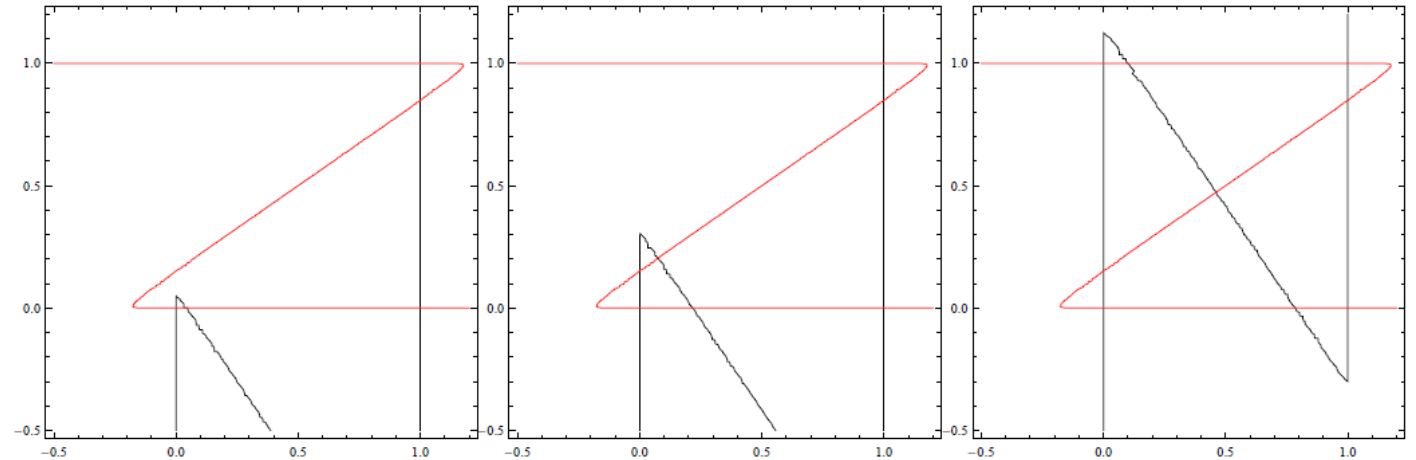

(a) (b)

Figure 12 (a) $\theta_{1}=0.5=\theta_{2} ;$ (b) $\theta_{1}=2, \theta_{2}=0.5 ;$ (c) $\theta_{1}=7, \theta_{2}=0.5$

Let $\mu=40, \gamma_{1}=\gamma_{2}=1$ and the entries of the matrix $W$ are $w_{11}=9, w_{12}=-6, w_{21}=2$ and $w_{22}=3$. 


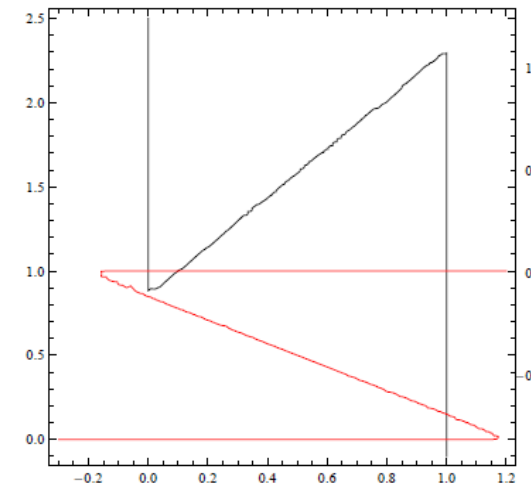

(a)

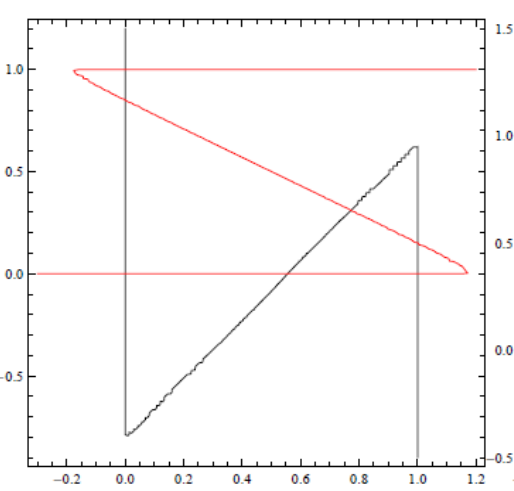

(b)

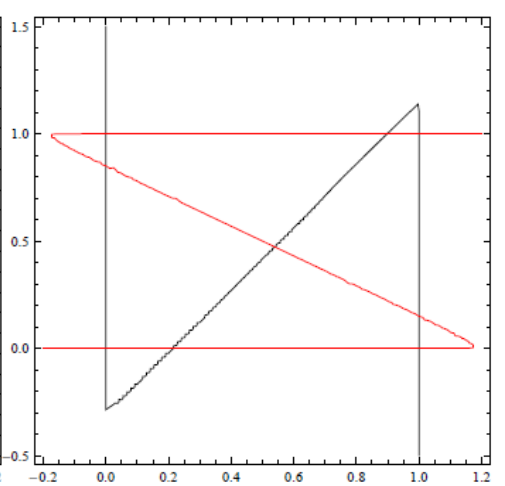

(c)

Figure 13 (a) $\theta_{1}=-5, \theta_{2}=2.5$; (b) $\theta_{1}=5, \theta_{2}=2.5$; (c) $\theta_{1}=2, \theta_{2}=2.5$

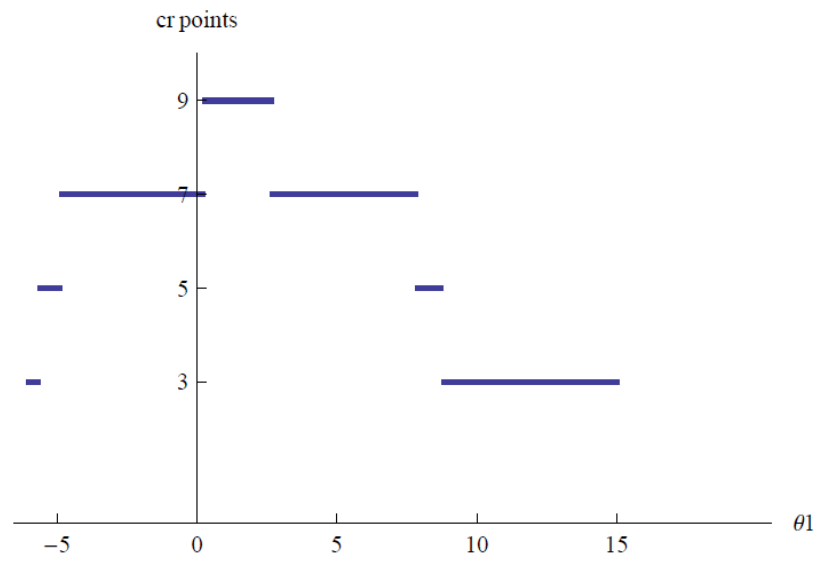

Figure 14 Parameters are $\mu=40, \gamma_{1}=\gamma_{2}=1, w_{11}=9$, $w_{12}=-6, w_{21}=2, w_{22}=3$. The graph shows the number of critical points for a given $\theta_{1}$.

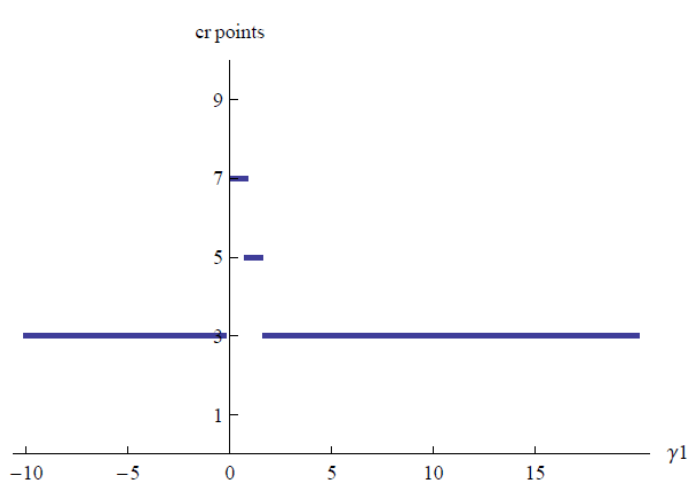

Figure 15 Parameters are $\mu=40, \gamma_{1}=\gamma_{2}=1, w_{11}=9$, $w_{12}=-6, w_{21}=2, w_{22}=3$. The graph shows the number of critical points for a given $\gamma_{1}$.

\section{Conclusions}

The systems (10) and (11) are the two-component models of GRN. These systems always have at least one critical point. For the regulatory matrices with zero entries in the main diagonal, the activation and the mixed cases can be geometrically analyzed. The activation systems can have attractors that consist of a single point or a couple of stable critical points. Any particular system can be geometrically analyzed by considering the nullclines and the vector fields. The influence of the change of parameters can be traced. In the mixed activation-inhibition case, the vector field rotates trajectories around the unique critical point which is a stable focus. Closed orbits are not possible. If the elements of the regulatory matrix $W$ on the main diagonal are not zeros, closed orbits are possible. Moreover, the number of critical points then can vary from one to nine. The shape of nullclines is changed substantially under the change of the parameters.

\section{References}

[1] Y. Koizumi. Adaptive Virtual Network Topology Control Based on Attractor Selection. Journal of Lightwave Technology. 2010; 28(11): 1720- 1731, doi: 10.1109/JLT.2010.2048412.

[2] https://en.wikipedia.org/wiki/Sigmoid_function.

[3] https://en.wikipedia.org/wiki/Gompertz_function.

[4] A. Spirov, D. Holloway. Using evolutionary computations to understand the design and evolution of gene and cell regulatory networks. Methods. 2013; 62(1): 3955, doi:10.1016/j.ymeth.2013.05.013.

[5] Yifei Wang. Review of Wagners Artificial Gene Regulatory Networks Model and its Applications for Understanding 
Complex Biological Systems. COJ Robotics \& Artificial Intelligence. COJRA.000501.2019; 1(1): 1-13.

[6] F.M. Alakwaa. Modeling of Gene Regulatory Networks: A Literature Review. J. Computational Systems Biology. 2014; 102, 1(1): 1-8.

[7] H. D. Jong. Modeling and Simulation of Genetic Regulatory Systems: A Literature Review. J. Computational Biology. 2002; 9(1): 67-103. doi: 10.1089/10665270252833208.

[8] T. Schlitt. Approaches to Modeling Gene Regulatory Networks: A Gentle Introduction. In: Silico Systems Biology. Methods in Molecular Biology (Methods and Protocols), Humana Press; 2013;1021:13-35. doi:10.1007/9781-62703-450-0.

[9] N. Vijesh, S. K. Chakrabarti, J. Sreekumar. Modeling of gene regulatory networks: A review. J. Biomedical Science and Engineering, 2013; $6: 223-231$.

[10] T. Kohsokabe, K. Kaneko. Boundary-induced pattern formation from uniform temporal oscillation. Chaos. 28, 045110 2018; doi: 10.1063/1.5013280.

[11] F. Sadyrbaev. Planar differential systems arising in network regulation theory. Advanced Mathematical Models \& Applications. 2019; 4 (1): 70-78.

[12] K.W. Kohn. Molecular interaction maps as information organizers and simulation guides. Chaos. 2001; 11 (1): 84-97, doi: $10.1063 / 1.1338126$.

[13] S. Atslega, D. Finaskins, F. Sadyrbaev. On a Planar Dynamical System Arising in the Network Control Theory. Mathematical Modelling and Analysis. 2016; 21 (3):385-398.

[14] E. Brokan, F. Sadyrbaev. On a differential system arising in the network control theory. Nonlinear Analysis: Modelling and Control, 2016; 21(5): 687-701, doi:10.15388/NA.2016.5.8.

[15] E. Brokan, F. Sadyrbaev. On Attracting Sets of a Certain Class of Differential Systems. Enlightenment of Pure and Applied Mathematics. 2016; 2(1): 153-159.

[16] D. Finaskins, E. Brokan, F. Sadyrbaev. Attracting Sets in Network Regulatory Theory. Advances in Wireless and Optical Communications (RTUWO), 2016, doi: 10.1109/RTUWO.2016.7821886.

[17] E. Brokan, F. Zh. Sadyrbaev. On Attractors in Gene Regulatory Systems. AIP Conference Proceedings. 1809(020010), 2017; doi: 10.1063/1.4975425.

[18] E. Brokan, F. Zh. Sadyrbaev. On Attractors in Gene Regulatory Systems. In: Proceedings of the APMAS 2016. AIP Conference Proceedings 1809, edited by A.Y. Oral et al., (020010), 2017. doi: 10.1063/1.4975425.

[19] E. Brokan, F. Sadyrbaev. Attraction in n-dimensional differential systems from network regulation theory. Mathematical Methods in Applied Sciences. 2018; 41(17):7498-7509 https://doi.org/10.1002/mma.5086.

[20] M. Marin. On the domain of influence in thermoelasticity of bodies with voids. Archivum Mathematicum. 1997; 33 (4): 301-308.

[21] M. Marin. An uniqueness result for body with voids in linear thermoelasticity. Rendiconti di Matematica, Serie VII Roma : 1997; 17: 103-113.

[22] M. Marin. An evolutionary equation in thermoelasticity of dipolar bodies Journal of Mathematical Physics 1999; 1391 (40) https://doi.org/10.1063/1.532809.

[23] S. Lefschetz. Differential equations: geometric theory. Interscience publ., 1957. 